\title{
Evaluation of the Soil Nutrients of Added Cultivated Land Based on Entropy Weight-Fuzzy Comprehensive Method: A Case Study in Chengcheng County, Shaanxi Province
}

\author{
Chendi Shi ${ }^{1,2,3}$, Juan $\mathrm{Li}^{1,2,3, \mathrm{a}, *}$ and Tingting $\mathrm{Cao}^{1,2,3}$ \\ ${ }^{1}$ Shaanxi Land Engineering Construction Group co., LTD, Xi'an, China. \\ ${ }^{2}$ Institute of Land Engineering Technology in Shaanxi Province co., LTD, Xi'an, China. \\ ${ }^{3}$ Key Laboratory of Degraded and Unused Land Remediation Engineering of Ministry of Land and \\ Resources, PRC, Xi'an, China. \\ *,agsqyscd@163.com
}

\begin{abstract}
In this paper, through the sampling analysis, the entropy weight---fuzzy comprehensive evaluation was adopted to distribute the weight of added cultivated land fertility in Taiyuan District of Weibei, and the soil fertility in the region was further evaluated. At the same time, the main limiting factor impacting soil fertility of the added cultivated land was put forward. The results indicate that 1) Indicators of soil fertility weight of newly-increased farmland in Taiyuan District of Weibei region are as follows: organic matter $20.19 \%$, total nitrogen $20.40 \%$, Olsen-P $33.56 \%$, and Olsen-K $25.85 \%$. 2) The soil fertility scores of these farmland in Chengcheng County under the land consolidation project are dispersed with values ranging from 4.80 to 20.96, with the mean of 11.80. 3) Available phosphorus (AP), soil organic matter (SOM) and available potassium (AK) are the major factors behind low soil fertility indexes. The extent of their impact on soil fertility is ranked as follows: $A P>A K>S O M .4) A P$ and $A K$ are the most important constraints which lead to low soil fertility of new farmland. The most effective way to improve the composite index of soil fertility is that the content of AK, AP and SOM in the soil should be increased successively. And priority should be given to AP and AK. 5) The adoption of a combination of Analytic Hierarchy Process and weight comparison method has yielded good results in evaluating soil fertility, which implies broad prospect of application.
\end{abstract}

Keywords: sampling analysis; comprehensive evaluation; comparison method.

\section{Introduction}

In recent years, China has adopted the policy of occupational and compensational balance to the cultivated land to meet the need of land resources during the process of urban development. Since the adoption of the policy, there is a marked increase in the scale of added cultivated land. While we strive to achieve equilibrium of the total number of cultivated land, the quality of added cultivated land has increasingly become a great concern to many people. Because there are a large number of earth piles in the land remediation process, which leads to soil remodeling and poor nutrients, the situation about soil nutrients are not optimistic. Therefore, the correct understanding and scientific and objective evaluation of soil nutrients constitutes a prerequisite for accurate understanding of soil nutrients in newly added cultivated land and better use of soil resources. It has also become a popular subject of research at home and abroad in recent years [1-5]. The examination and understanding of the basic situation of soil nutrients in added cultivated land after land remediation and the pinpointing of the areas of low content of soil nutrients have exerted a far-reaching significance to improve the soil quality of added cultivated land in this area.

The evaluation of cultivated land quality is of great importance in guiding agricultural production. The success or failure of farmland quality assessment methods directly affects the reliability of farmland quality assessment results. There are a variety of methods for evaluating soil nutrients in added cultivated land. As a result, the evaluation results will be different because of different evaluation methods adopted. At present, there is no uniform standard for the evaluation of soil nutrient indicators of added cultivated land. A systematic and comprehensive evaluation method for the quality of land projects is a method formulated by the Ministry of Land and Resources to grade and 
assess agricultural land quality across China. The method, mainly based on the calculation of the productive potential of cultivated land, assesses and grades the quality of cultivated land from both natural and economic conditions. Currently, the main evaluation methods frequently used are AHP [9], fuzzy comprehensive evaluation method [10], comprehensive index method [11], principal component analysis method [12], fuzzy neural network model method [13], etc. The entropy--fuzzy comprehensive evaluation method in the evaluation of cultivated land quality has not been reported yet. Researches on land remediation projects both at and broad disproportionately focus on the model of land remediation projects [14] and evaluation of integrated effect after land remediation [15-17]. Meanwhile, researches on soil nutrient assessment also focus on high-quality farmland [18, 19]. Few researches are conducted to study and assess soil nutrient of added cultivated land featured by low content of soil nutrients [20-21]. Nor has the study of short board effect of soil fertility in added cultivated land been reported. This paper adopts the entropy-fuzzy comprehensive evaluation method to conduct a comparative analysis of the weights of soil nutrients in newly added cultivated land in Taiyuan District of Weibei, and then calculates the comprehensive index of soil nutrients in newly added cultivated land. At the same time, the maximum limiting factor of soil nutrients in newly added cultivated land was explored in order to provide a basis for the evaluation of new soil nutrients and fertilization management in the area surveyed.

\section{Materials and Methods}

\subsection{An Overview of the Area Surveyed}

The land remediation project of Chengcheng County, Taiyuan District of Weibei region was selected as the area for study. Chengcheng County is located in the east of Shaanxi Province. The geographic coordinates are $109^{\circ} 46^{\prime} 30^{\circ} \mathrm{E}$ to $110^{\circ} 05^{\prime} 50^{\circ} \mathrm{E}$, and $34^{\circ} 55^{\prime} 45^{\circ} \mathrm{N}$ to $35^{\circ} 27^{\prime} 05^{\circ} \mathrm{N}$. The terrain is high in the north and low in the south, and four rivers divide Chengcheng County into a place featured by "three beams and one plain". The county is located in the Guanzhong Plain and belongs to the warm temperate monsoon zone, with an average annual temperature of $12^{\circ} \mathrm{C}$, rainfall of 680 $\mathrm{mm}$ and frost-free period of 204 days. This area is also featured by large temperature difference between day and night. The annual hours of sunshine reach $2616 \mathrm{~h}$ and the altitude is 406.9 to 1268 meters above the sea level.

\subsection{Sample Collecting and Processing}

Through field trips, 45 project areas for new arable land were selected for sampling in the research area in March 2015. The research area occupies an area of about $560 \mathrm{hm} 2$, and each project area is about $10 \mathrm{hm} 2$. Snake-shaped distribution method was adopted. Three sampling points were stationed in each project area. Every sampling point consisted of 0-30 $\mathrm{cm}$ topsoil gathered in the central part and four corners of the sampling place. Then 5-point mixing method was adopted. After mixed, the samples were controlled to $2 \mathrm{~kg}$ and composed of 135 sampling points. The soil samples were taken to the lab and air-dried for later use. Due to small differences in environmental conditions and spatial variability, in addition to the same soil types of the project areas and given that all the project areas were deserted grasslands before the remediation, the spatial variability of soil nutrients was small. Therefore, the number of samples can basically represent the actual soil nutrients in the project area.

When the samples air-dried, they will be possessed in the lab. Indicators of soil $\mathrm{pH}$, organic matter (SOM) [22], total nitrogen (TN) [23], available phosphorus (AP) and available potassium (AK) [24] were measured. The entropy weight--fuzzy comprehensive evaluation method [9] was adopted to determine the weight of soil nutrients in new cultivated land, and the circumstances of soil nutrients in the newly added cultivated land, rich or poor, were analyzed through comparison of weights. 


\subsection{Evaluation Model Building}

\subsubsection{The Building of Assessment System}

According to the soil nutrient evaluation index and the soil nutrient index in the research area, the soil nutrient index system selected in this paper consists of four indicators, including soil organic matter, soil total nitrogen, soil available phosphorus, and soil available potassium.

\subsubsection{Determination of the Weight of Fuzzy Evaluation Factors Based on Entropy Method}

The information obtained in assessing decisions is one of the decisive factors evaluating precision and reliability. In information theory, entropy is a measure of the degree of disorderly system. It can also measure the amount of information provided by the data. When entropy is used to determine the weight, the value of the evaluation object on a certain index varies greatly, and the entropy is smaller. It shows that the amount of effective information provided by this indicator is larger, and the weight of this indicator should also be larger; conversely, if the difference between the value of an indicator is smaller, the entropy value is larger, indicating that the amount of information provided by the indicator is small, and the indicator's weight should also be small. When the value of each object under evaluation is exactly the same in a certain index, the entropy value reaches the maximum, which means that the index does not provide any useful information for the decision, and it can be removed from the evaluation index system. Therefore, entropy method is an objective weighting method. In the evaluation of land resources, the weight is determined by calculating the entropy, which is to determine the weight of each evaluation index according to the degree of difference in each evaluation index value. Suppose there are $m$ evaluation indicators and $n$ objects of evaluation, matrix mathematical formula $X=\left(x_{i j}\right)_{m \times n}$ from the original data is established. As to a certain index i, if there is a larger difference among the scale values of xij, the index will play a stronger role in the evaluation. If the scale values of an index are all equal, the index will has little effect in the comprehensive evaluation. The use of entropy weighting includes the following three steps:

1. Standardize

the matrix of original data Suppose m evaluation indicators and $n$ objects of evaluation, the matrix of original data is formed:

$$
X=\left(\begin{array}{ccc}
x_{11} & \cdots & x_{1 n} \\
\vdots & & \vdots \\
x_{m 1} & \cdots & x_{m n}
\end{array}\right)
$$

Standardize the matrix, we can get

$$
R=\left(r_{i j}\right)_{m \times n}
$$

In the equation $r_{i j}$ - the standard value $r_{i j} \in[0,1]$ of the $\mathrm{j}$-th evaluation object on the $\mathrm{i}$-th evaluation index. For indicators that indicates bigger ones are superior:

$$
r_{i j}=\frac{x_{i j}-\min _{j}\left\{x_{i j}\right\}}{\max _{\mathrm{j}}\left\{x_{i j}\right\}-\min _{j}\left\{x_{i j}\right\}}
$$

For indicators that indicates smaller ones are superior

2. Define the entropy

$$
r_{i j}=\frac{\max _{\mathrm{j}}\left\{x_{i j}\right\}-x_{i j}}{\max _{\mathrm{j}}\left\{x_{i j}\right\}-\min _{j}\left\{x_{i j}\right\}}
$$

In the evaluation question of $m$ evaluation indexes and $n$ evaluation objects, the entropy of the ith index is defined as

$$
H_{i}=-k \sum_{j=1}^{n} f_{i j} \operatorname{Inf}_{i j}, \quad i=1,2,3, \cdots, m
$$


In the equation $f_{i j}=r_{i j} / \sum_{j=1}^{n} r_{i j}, k=1 / \ln n$, when $f_{i j}=0, f_{i j} \operatorname{In} f_{i j}=0$

3. Define the weight of the entropy

After defining the entropy of the i-th index, the entropy weight of the i-th index is defined as:

$$
\mathrm{w}_{i}=\frac{1-H_{i}}{m-\sum_{i=1}^{m} H_{i}}
$$

In the equation, $0 \leq w_{i} \leq 1$, at the same time $\sum_{i=1}^{m} w_{i}=1$

From the foregoing analysis, the entropy weight method is a mathematical method for calculating a comprehensive index based on integrated consideration of the information provided by each evaluation index. The entropy is not the index which reflects the importance and weight of evaluation indicators in real sense concerning the evaluation of the decision-making problem, but rather represents the degree of comparative intensity among indicators under the condition that the values of all indicators are definitive after the object set is given. From the perspective of information, it represents the extent to which the evaluation indicator provides effective information in solving the problem. As an objective and comprehensive evaluation method, it mainly determines the weight based on the amount of information that each indicator passes to the decision maker.

Table 1. Soil Nutrient Weights of Added Cultivated Land

\begin{tabular}{ccccc}
\hline Index & SOM & TN & AP & AK \\
\hline Weight $(\%)$ & 0.2019 & 0.2040 & 0.3356 & 0.2585 \\
\hline
\end{tabular}

The weights of each evaluation index obtained by the entropy weight method are shown in Table 1. The weight of soil available phosphorus is the largest. This may be due to the great spatial difference in soil available phosphorus in the Taiyuan District of Weibei region.

\section{Grading of Evaluation Index}

We adopt the grading standard of soil nutrients formulated in the second national soil survey, which includes six levels: enormously rich, rich, medium, poor, very poor and desperately poor.

\subsection{Establishment of Set the Appraisal}

The division of evaluation results requires the use of a appraisal set, which is a set of evaluation criteria for the indicator. Each evaluation index is graded and divided into six levels of U1, U2, U3, $\mathrm{U} 4$, U5 and U6, corresponding to the natural language of enormously rich, rich, medium, poor, very poor and desperately poor. Suppose the appraisal set $U=\{U 1, U 2, U 3, U 4, U 5, U 6\}, \quad(i=1,2,3,4,5$, 6) represents the i-th evaluation level.

\subsection{Determination of the Degree of Membership}

For any set $\mathrm{x}$ in the research scope $\mathrm{U}$, if we can always find a number which can correspond with $\mathrm{x}$, then $\mathrm{B}$ is the fuzzy set in $\mathrm{U}$ and $\mathrm{B}(\mathrm{x})$ is called the degree of membership of $\mathrm{x}$ on A. Membership function is the foundation for the application of fuzzy control.

For the soil nutrient index, the degree of membership of the measured value of the index between Level 1 and 6 is calculated by Equation 4.

$$
\mathrm{b}_{i j}= \begin{cases}0 & , \quad x_{i j} \leqslant y_{i 6} \\ \frac{x_{i j}-y_{i 6}}{y_{i 1-} y_{i 6}} & , \quad y_{i 1}>x_{i j}>y_{i 6} \\ 1 & , \quad x_{i j} \geqslant y_{i 1}\end{cases}
$$


In Equation 4, bij is the relative membership degree of the eigenvalue of index $\mathrm{i}$ in Sample $\mathrm{j}$ to fuzzy concept A. The modular concept A here is "rich", i.e., the relative membership degree to "rich" of standard value of the level 1 greater than or equal to the index $i$ is 1 ; the relative subordination to "rich" of standard value of the level 6 less than or equal to the index i is 0 ; yi1, yi6 are the 1st and 6th standard values of the index i respectively.

Construct a mathematical formula for the membership degree of the suboptimal degree $\bar{R}_{m n}$ according to Equation 4.

$$
\bar{R}_{m n}=\left[\begin{array}{cccc}
0.05 & 0.27 & 0.06 & 0.11 \\
0.00 & 0.54 & 0.00 & 0.09 \\
\ldots & \ldots & \ldots & \ldots \\
0.11 & 0.24 & 0.08 & 0.20 \\
0.12 & 0.23 & 0.06 & 0.17
\end{array}\right]
$$

\subsection{Analysis of Evaluation Results}

After calculating the degree of membership, we have obtained the index scores of soil nutrient in Chengcheng County, as shown in Figure 1.

Because different factors of influence have distinct impact on and make differentiated contribution to soil nutrients, we determine the indexes weight of soil nutrients by way of information entropy.

According to the scores (Si) and weights Wi of different levels of each index, the following evaluation model can be established:

$$
C=\sum^{\mathrm{n}} S_{\mathrm{i}} \circ W_{\mathrm{i}} \quad(i=1,2,3, \cdots, 7)
$$

Among them, $\mathrm{C}$ is the comprehensive $5 \mathrm{se}$ ore value for evaluating soil nutrients in added cultivated land. According to the previous definition of score value and weight, $\mathrm{C}$ value will also be between $[0,6]$. Si is the score of the $\mathrm{i}$-th factor, $\mathrm{Wi}$ is the weight value of the $\mathrm{i}$-th factor and $\mathrm{n}$ represents the number of evaluation factors. Finally, the value will be converted within $[0,100]$

\section{Evaluation Results}

\subsection{Distribution Characteristics of Soil Nutrient Index of New cultivated Land}

Based on the Si value and Wi value of the above evaluation factors, the comprehensive evaluation index of soil nutrients in each project area was obtained by using the evaluation model constructed. The distribution of fertility index in newly added cultivated land of each project area is shown in Figure 1.

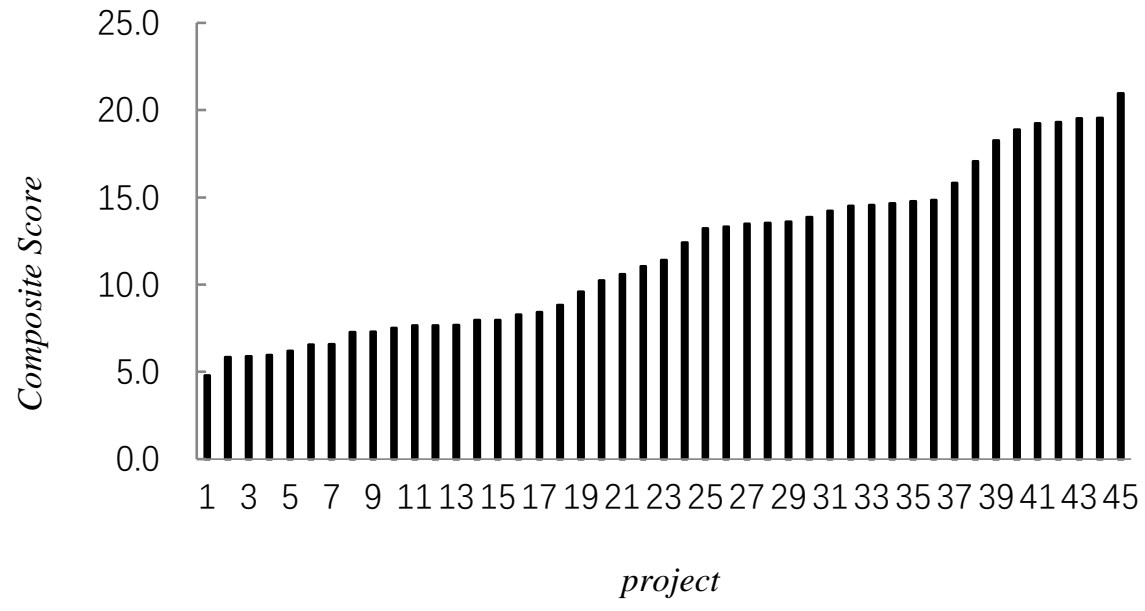

Figure 1. The score range of comprehensive index of soil nutrient for each project 


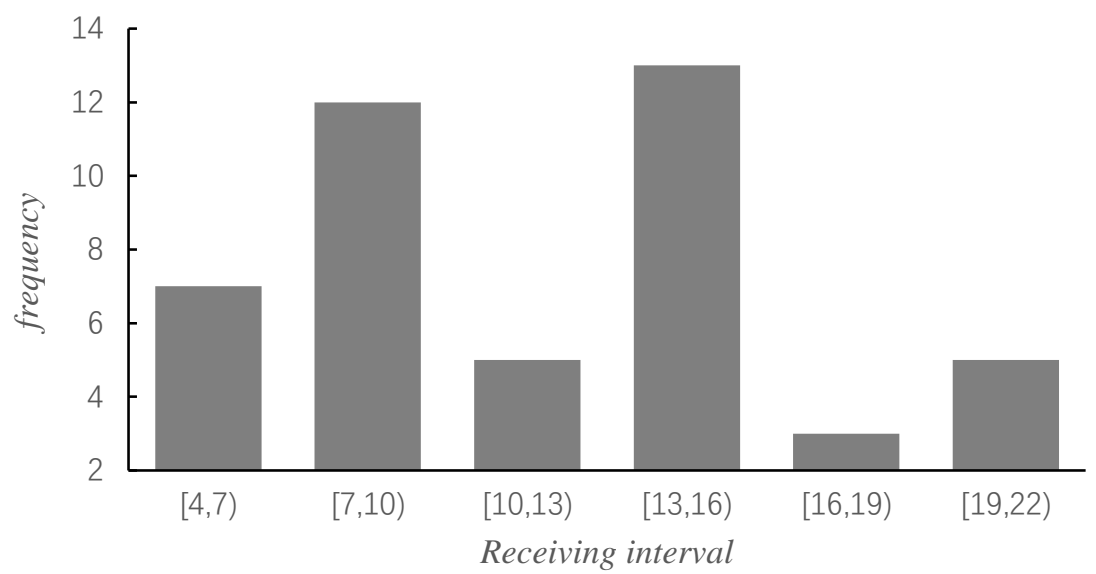

Figure 2. Frequency Histogram of Fertility Value and the Number of Project Area for New Cultivated Land

According to the evaluation results (Figure 2), the scores of soil nutrients of added cultivated land in each project area are distributed between 4 and 22. The lowest score is 4.80 , the highest one is 20.96 , and the average is 11.80 , with an average deviation of 4.62 . The area of highest distributed scores ranges from 13 to 16 points, covering 13 project areas accounting for about 1/3 of the project area. And it is followed by 7 to 10 points, covering 12 project areas, nearly $1 / 3$ of the total. The distribution area of the lowest score is 16 to 19 points, covering only 3 project areas accounting for about $6.67 \%$ of the total number. A total of 21 projects with scores greater than 13 account for $46.67 \%$ of the total project areas. There are only 24 project areas with scores less than 13 .

\subsection{Level Distribution of Soil Nutrients in New Cultivated Land}

In reference to the comprehensive grading standard, 0 to 100 points are divided into five levels, that is, 80 to 100 points is classified as Level 1 cultivated land, 60 to 80 is classified as Level 2 cultivated land, 40 to 60 is classified as Level 3 cultivated land, 30 to 50 is classified as Level 4 arable land, and 0 to 30 is divided into Level 5 arable land. We can see that soil nutrients of cultivated land added for land remediation project in Taiyuan District of Weibei are dispersed within Level 2, Level 3 and Level 4. The statistical results of each indicator are shown in Table 2.

Table 2. The Soil Nutrient Indicators at Various Levels

\begin{tabular}{cccccc}
\hline Grade & $\begin{array}{c}\text { Organic matter } \\
(\mathrm{g} / \mathrm{kg})\end{array}$ & $\begin{array}{c}\text { Total nitrogen } \\
(\mathrm{g} / \mathrm{kg})\end{array}$ & $\begin{array}{c}\text { Available } \\
\text { phosphorus } \\
(\mathrm{mg} / \mathrm{kg})\end{array}$ & $\begin{array}{c}\text { Available } \\
\text { potassium } \\
(\mathrm{mg} / \mathrm{kg})\end{array}$ & $\begin{array}{c}\text { The number } \\
\text { of sampling } \\
\text { points }\end{array}$ \\
\hline Level 2 & $10.90 \pm 0.66$ & $0.99 \pm 0.25$ & $5.21 \pm 1.91$ & $64 \pm 12$ & 5 \\
Level 3 & $8.72 \pm 2.24$ & $0.95 \pm 0.23$ & $4.05 \pm 1.54$ & $55 \pm 13$ & 28 \\
Level 4 & $4.46 \pm 2.80$ & $0.87 \pm 0.24$ & $3.61 \pm 1.23$ & $41 \pm 10$ & 12 \\
Mean value & $7.82 \pm 3.12$ & $0.93 \pm 0.23$ & $4.06 \pm 1.54$ & $52 \pm 14$ & 45 \\
\hline
\end{tabular}

\subsection{Analysis of Limiting Factors of Soil Fertility in Added Cultivated Land}

The weight value of each index determined by the entropy method is called the theoretical weight, and the actual weight is obtained according to the proportion of the weight of the soil level corresponding to the measured data of the soil sample. The actual weight results reflect the actual ratio of soil nutrients in the current research area. By analyzing the theoretical weights and actual weights of soil nutrients in the study area (Table 3), it can be seen that the actual weights of available phosphorus, available potassium, organic matter, and total nitrogen. Based on barrel-effect theory and using weight contrast analysis method, we can see that the main cause of low soil nutrient index is the lower content of soil available phosphorus and available potassium (Figure 3). The impact 
degree is $\mathrm{AP}>\mathrm{AK}>\mathrm{SOM}>\mathrm{TN}$. Among them, soil available phosphorus and available potassium are the two main limiting factors that cause deficient soil nutrients under cultivated land. It can be seen that the most effective way to increase the comprehensive index of soil nutrients is to increase AP, $\mathrm{AK}, \mathrm{SOM}$, and TN in turn, and the priority should be given to increase the content of available phosphorus and available potassium in the soil.

Table 3. Comparison of Theoretical Weight and Actual Weight of Soil Nutrients in Added Cultivated Land

\begin{tabular}{ccccc}
\hline Index & SOM & TN & AP & AK \\
\hline Theoretical weight (\%) & 20.19 & 20.40 & 33.56 & 25.85 \\
Actual weight (\%) & 14.62 & 21.41 & 9.05 & 7.67 \\
\hline
\end{tabular}

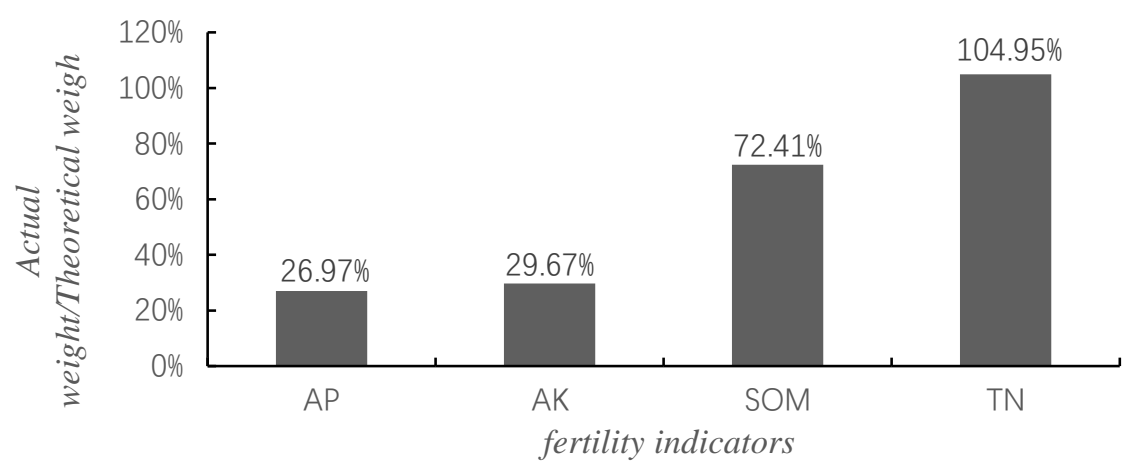

Figure 3. Distribution of Weight Proportion of Various Fertility Indicators

\section{Discussion}

In this paper, four indicators that have an impact on soil nutrients are selected, namely SOM, AP, AK, and TN. Among them, SOM, AP, AK, and TN have good comparability as conventional soil indicators, and they have important influence on the growth of corps. This paper uses entropy weightfuzzy comprehensive evaluation method to analyze the weight of evaluation indicators, and conducts a consistency test. The distribution of weight are similar to results obtained by Wu Yuhong [29] through the principal component analysis method. The results can serve as a reference for evaluation of soil nutrient indicators in Taiyuan District and even in Guanzhong Region of Central Shaanxi. As a consequence, this paper can reflect the hallmark of soil nutrients of added cultivated land.

The weight distribution of various fertility indexes indicates that the reason for the low comprehensive soil index mainly depends on the lower content of AP, AK and SOM in the added arable land than the normal level. In particular, AP and AK are the two main limiting factors for low soil nutrient comprehensive index in added farmland. The research conducted by Li Like [30] in the 1980s shows that phosphorus deficiency is the main limiting factor to high yield of wheat in the Weibei Highland. And this is supported by Li Ru's research recently [31]. This is consistent with the findings of this article. This fact also shows that the weight assigned based on the AHP in this region is correct. It has a strong reference value. The reason for this result may be that during the land remediation process, the underlying primary soil forms new topsoil, resulting in the lack of phosphorus in the soil, due to the huge disturbance or even reconstruction of the soil. There are two solutions to this problem. One is to do a good job of backfilling the topsoil in the land remediation process. Second, after land consolidation is completed, the comprehensive index of soil fertility in the region will be increased by increasing farmyard manure and chemical fertilizers (especially phosphate fertilizer and potash fertilizer). This article has completed the necessary ordering of soil nutrient replenishment, but further research is needed on the rapid maturation of soil in added arable land and precise soil fertilization in specific regions. 


\section{Conclusion}

1). Using the method of entropy weight, this paper concludes that the index of soil nutrient of added cultivated land in Chengcheng County's Land Remediation Project is 0.2019 for organic matter, 0.2040 for total nitrogen, 0.3356 for available phosphorus, and 0.2585 for available potassium.

2). The soil nutrients in added cultivated land of Chengcheng County remains the low and medium level and there is tremendously large room for improving the soil fertility.

3 ). The main factor that accounts for the low soil nutrient index of cultivated land in Chengcheng County is the lack of soil available phosphorus, available potassium, and organic matter.

4). Entropy weight method has achieved good results in soil nutrient evaluation and has a broad application prospect.

\section{Acknowledgments}

This work was financially supported by the Scientific Research Project of Engineering Construction Group Internal in Shaanxi Province fund (DNJY2017-20).

\section{References}

[1]. Zhang Shichao, Wei Chaofu, Li Ping. Analysis of potentialities and contribution rate of newlyincreased cultivated land from land development and consolidation $[\mathrm{J}]$. Transactions of the CSAE, 2010, 26(Supp.2): 312-319.

[2]. Yang Jin, Liu Li, Sun Chuanmin, et al. Soil components and fertility improvement of added cultivated land $[\mathrm{J}]$. Transactions of the CSAE, 2008,24(7): 102-105.

[3]. Gao Mingxiu, Li Zhanjun, Zhao Gengxing. Evaluation of land consolidation oriented cultivated land quality at project scale[J].Transactions of the CSAE, 2008,24(Supp.1): 128-132.

[4]. Sklenicka P. Applying evaluation criteria for the land consolidation effect to three contrasting study areas in the Czech Republic [J]. Land Use Policy, 2006, 23(4): 502-510.

[5]. Lichtenberg E, Ding C. Assessing farmland protection policy in china [J]. Land Use Policy, 2008, 25(1): 59-68

[6]. Yu Dongshen, Zhang Guangxing, Zhang Zhongqi, etal. Comparative study of bil-norm and norm for evaluating cultivated land quality [J]. Journal of soil, 2011, 48(2): 238-245.

[7]. Qu Yanbo, Zhang Fengrong, Guo Lina, et al. Estimation of farmland quality after rural residential land consolidation and its application [J].Transactions of the CSAE, 2012, 28 (2):226-233.

[8]. Ma Jianhui, Wu Ke ning, Zhao Huafu, et al. research on the dynamic cultivated land quality monitoring system based on the agricultural land gradation [J]. Chinese Agricultrural Resoures \& Regional Planning, 2013, 34(5): 133-139.

[9]. Liu Shuyi, Yang Qingyuan, He Chunyan, et al. The Benefit Assessment of Land Consolidation Based on Analytical Hierarchy Process (AHP) and Fuzzy Synthetic Evaluation-Case of 26 Villages in Chongqing [J]. Chinese Agricultural Science Bulletin, 2013, 29(26): 54-60.

[10]. Zhan Wei, He Liheng, Jin Xiaobin, et al. Performance evaluation for land consolation project using fuzzy comprehensive assessment method [J]. Journal of Nanjing Forestry University (Natural Science Edition), 2009, 33(2): 145-148.

[11]. Zhan Jiandong, Fan Zhou, Lai Jianqing et al. Application of comprehensive index quality evaluation model of soil heavy metal pollution in Taiyuan [J]. Research of Soil and Water Conservation, 2007, 14(4): 241-243. 
[12]. Xia Jianguo, Li Tingxuan, Deng Liangji, et al. The application of the principal component analysis method in quality evaluation of cultivated land [J]. Southwest China Journal of Agricultural Sciences, 2000, 13(2): 51-55.

[13]. Yang Xianye, Fu Qiang, Wang Baohua, et al. Construction and Application of Fuzzy Neural Networks Model in Evaluation on the Soil Quality Changes of SaCion engineering model in the plain round the Poyang Lake [J]. Chinese Journal of Agricultural resources and regional Planning, 2014, 35(01): 102-108.

[14]. Yang Jun, Wang Zhanxi, Jin Gui, et al. Post-benefit evaluation of land consolidation projective implementation based on ahp and fuzzy comprehensive evalution $[\mathrm{J}]$. Resources and environment in the Yangtze basin. 2013, 22 (08): 1036-1042.

[15]. He Ruhai, Nie Lei, He Fang. Evalution of the comprehensive benefits of the ecological conservation land consolidation: An example of Guichi area project in Chizhou,Anhui Province[J]. Journal of vhina Agricultural University, 2013, 18(4): 232-2371

[16]. Liu Ruiqing, Li Xinwang, Zhang Lulu, et al. Research of Sequence Structure on Comprehensive Benefit Evaluation of Soil Renovation [J]. Chinese Jaunal of Soil Sicence, 2012, 43(6): 1305-1310.

[17]. Wang Decai, Chang Qingrui, Liu Jing, et al. Evalution of soil fertility in Shaanxi based on soil space database [J]. Journal of Northwest A\&F University, 2008, 36(11): 105-110.

[18]. Fang Ruihong, Chang Qingrui, et al. Spatial variability and controlling factors of soil trace elements in hilly and gully area of the Loess Plateau [J]. Agricultural Research in the Arid Areas, 2012, 30(01): 25-29+42

[19]. Ma Chaoqun, Liu Tieming, Yang Meihuan. Analysis of soil nutrients of the newly-increased farmland in the process of land consolidation in Gaoling County [J]. Journal of Northwest A\&F University, 2010, 38(5): 175-18.

[20]. Ma Chaoqun, Chen Guixian, Wang Lixia. Analysis of soil nutrients content of newlyincreased farmland in the process of land consolidation in Lintong District $[\mathrm{J}]$. Agricultural Research in the Arid Areas, 2012, 30(2):47-51+61.

[21]. Ma Junyong, Cao Caiyun, Zhen Chunlian, et al. Effect of Long-term application of chemical fertilizers and organic manure on soil organic carbon and bulk density[J]. Chinese journal of soil and fertilizer, 2010, (6): 38-42.

[22]. Wang Juan, Lv Jialong, Xu Minggang, et al. The variation characteristics of nitrogen in red soil under long-term different fertilization [J]. Chinese journal of soil and fertilizer, 2010, (1): 16.

[23]. Zhang Beier, Huang Biao, Zhang Xiaoguang, et al. Evaluation of Soil Fertility Quality Variation During Past 30 Years in a Major Grain-producing Region of the North China Plain—A Case Study of Yucheng County [J]. Soils , 2012, 44 (3): 381-388

[24]. National soil survey office. Provisional technical regulations for the second national soil survey [M]. Beijing, Agricultural Press.1979

[25]. Long Hualou, Cai Yunlong, Wan jun. Sustainability Evaluation of Land Use in Development Zone: the Case of Kunshan [J]. Acta Geographica Sinica, 2000, 55(6): 719-728.

[26]. Xu Jianhua. Mathematical methods in modern geography [M]. Beijing, Advanced Education Press, 2002. 224-250. 
[27]. Su Shaoqing, Lin Bishan, Chen Zhengyi, et al. Comprehensive benefit evalution based on AHP — a case study of yunfu city, Guangdong Province[J]. South Country Journal, 2011, 1: 2226.

[28]. Wu Yuhong, Tian Xiaohong, Tong Yanan, et al. Assessment of integrated soil fertility index based on principal components analysis [J]. Chinese Journal of Ecology, 2010, 29(1): 173-180.

[29]. Li Like, Tian Jiaju, Gao Hua, et al. Soli phosphorus deficiency is an important factor limiting wheat yield in the Weibei dry tableland [J]. Shaanxi Journal of Agricultural Sciences, 1982(1): $33-34+25$.

[30]. Li Ru, Shan Yan, Li Shuili, et al. Analysis of Soil Fertility and Fertilization of Wheat Field in Shannxi [J]. Journal of Triticeae Crops, 2015, 35(1): 105-110. 